\title{
What Have We Learned from Perfusion MRI in Multiple Sclerosis?
}

\author{
(D)E. Lapointe, DD.K.B. Li, (D) A.L. Traboulsee, and (D) A. Rauscher
}

\begin{abstract}
SUMMARY: Using MR imaging, perfusion can be assessed either by dynamic susceptibility contrast MR imaging or arterial spin-labeling. Alterations of cerebral perfusion have repeatedly been described in multiple sclerosis compared with healthy controls. Acute lesions exhibit relative hyperperfusion in comparison with normal-appearing white matter, a finding mostly attributed to inflammation in this stage of lesion development. In contrast, normal-appearing white and gray matter of patients with MS has been mostly found to be hypoperfused compared with controls, and correlations with cognitive impairment as well as fatigue in multiple sclerosis have been described. Mitochondrial failure, axonal degeneration, and vascular dysfunction have been hypothesized to underlie the perfusion MR imaging findings. Clinically, perfusion MR imaging could allow earlier detection of the acute focal inflammatory changes underlying relapses and new lesions, and could constitute a marker for cognitive dysfunction in MS. Nevertheless, the clinical relevance and pathogenesis of the brain perfusion changes in MS remain to be clarified.
\end{abstract}

ABBREVIATIONS: ASL = arterial spin-labeling; DCE = dynamic contrast-enhanced; NAWM = normal-appearing white matter; RRMS = relapsing-remitting MS

M ultiple sclerosis is considered an immune-mediated disease characterized by inflammation and neurodegeneration. ${ }^{1}$ However, the pathogenesis of the disease is incompletely understood. ${ }^{1}$ Advanced imaging techniques have been used to better characterize and understand the disease. Interest in vascular pathology contributing to MS pathogenesis has grown following several observations: Lesions are known to predominantly develop around small central veins, ${ }^{2}$ and there is lymphocytic infiltration of the vein walls, perhaps preceding perivenular inflammatory infiltration. ${ }^{2,3}$ Microvascular occlusive changes have occasionally been described, suggesting that ischemia could occur in MS. ${ }^{4}$

Positron-emission tomography and single-photon emission CT were first used to evaluate brain perfusion in MS. Early studies found white and gray matter oxygen hypometabolism and reduced blood flow in comparison with healthy controls, with some correlations with cognitive dysfunction. ${ }^{5-7} \mathrm{MR}$ imaging is a non-

From the Division of Neurology (E.L., A.L.T.), Department of Medicine (E.L., A.L.T.), MRI Research Center (A.R.), and Departments of Pediatrics (A.R.) and Radiology (D.K.B.L.), University of British Columbia, Djavad Mowafaghian Center for Brain Health, Vancouver, British Columbia, Canada.

Please address correspondence to Emmanuelle Lapointe, MD, Division of Neurology and Department of Medicine, DM Center for Brain Health, 2215 Wes-

brook Mall, Vancouver, BC, V6T 2B5 Canada; e-mail:

emmanuelle.lapointe@usherbrooke.ca

- Indicates open access to non-subscribers at www.ajnr.org

http://dx.doi.org/10.3174/ajnr.A5504 ionizing radiation technique that offers faster acquisition and better signal-to-noise and contrast-to-noise ratios than these techniques, and it allows unlimited repeat measurements in the same patients. $^{8}$

This review summarizes the perfusion MR imaging literature in MS, important clinical correlations, and presumed underlying mechanisms.

\section{Perfusion MR Imaging Techniques}

Using MR imaging, perfusion can be assessed by either measuring the effects of a paramagnetic contrast agent or labeling inflowing spins with a radiofrequency pulse. The former approach includes dynamic susceptibility contrast and dynamic contrast-enhanced (DCE) MR imaging; the latter is called arterial spin-labeling (ASL).

\section{Dynamic Susceptibility Contrast MR Imaging}

In DSC, an intravenous paramagnetic contrast agent is administered, and the brain is scanned with a rapid imaging technique, typically $\mathrm{T} 2{ }^{\star}$-weighted single-shot gradient-echo-planar imaging $^{9}$ or a T2-weighted spin-echo-planar scan. When the contrast agent reaches the tissue vasculature, it makes the vessels more paramagnetic, creating field inhomogeneities around the vessels. These lead to accelerated dephasing of magnetization (ie, a signal reduction via accelerated $\mathrm{R} 2^{\star}$ relaxation). By measuring the signal dynamically with a temporal resolution of typically $1-2$ seconds and knowing the relaxivity of the contrast agent, one can measure 
the contrast agent concentration across time. This function can be converted into maps of cerebral blood flow, cerebral blood volume, and temporal parameters such as mean transit time. ${ }^{9}$ However, this step requires a deconvolution with the concentration function in a feeding artery, the arterial input function, which has its limitations. Deconvolution is an inherently ill-posed mathematic operation, and accurate measurement of the arterial input function is hampered by partial volume effects. ${ }^{9}$

A variant of gradient-echo DSC is spin-echo DSC, which uses a spin-echo-planar scan. The effects of a contrast agent on the spin-echo signal are much weaker than on the gradient-echo signal. In the former, the signal loss is mainly mediated by diffusion of spins within a magnetically inhomogeneous environment. In the latter, there is additional static dephasing of spins in the same inhomogeneous environment. Due to the stronger effect of the contrast agent on the gradient-echo signal, most studies use gradient-echo signal planar imaging. Numerical simulations ${ }^{10,11}$ and experiments ${ }^{10}$ have shown that spin-echo DSC is particularly sensitive to capillary-sized vessels, whereas the sensitivity of gradient-echo DSC is similar for a broad range of vessel sizes. Both techniques benefit from higher field strengths due to increased susceptibility effects from the contrast agent.

Typical parameters of a gradient-echo DSC scan are a voxel size of $3 \times 3 \mathrm{~mm}$ and a slice thickness of $3-5 \mathrm{~mm}$. At 3T, the TE is in a range of 30-50 ms for gradient-echo DSC and around 50-70 ms for spin-echo DSC. The TR is as short as possible, typically between 1 and 2 seconds. Because the DSC scan needs to capture the dynamics of the contrast agent entering and exiting the brain tissue, scan times are typically in the range of $1-3$ minutes. ${ }^{8,12,13}$ The cortical ribbon is thinner than the typical voxel size of DSC MR imaging. Recent developments in rapid imaging using multiband MR imaging ${ }^{14}$ will allow further reduction of TR and/or reduction of voxel size. The reduced TR increases the temporal signal-to-noise ratio, ${ }^{15}$ and the smaller voxel size reduces partial volume effects between cortical gray matter and underlying white matter, allowing improved assessment of cortical perfusion.

\section{Dynamic Contrast-Enhanced Imaging}

Dynamic contrast-enhanced imaging uses T1-shortening due to a contrast agent ${ }^{16}$ to detect areas of disrupted blood-brain barrier. DCE-MR imaging is performed by acquiring repeat T1-weighted scans after intravenous injection of a contrast agent. The signal enhancement across time can be used to compute maps of bloodbrain barrier integrity. Therefore, DCE is the standard approach for the measurement of permeability. However, high-temporalresolution DCE can also assess perfusion information. DCE is mainly used for tumor imaging, and applications to MS are rare though it has been used to characterize the spatiotemporal enhancement patterns of active lesions. ${ }^{17}$ Typical scan times are in the range of $4-8$ minutes. ${ }^{17}$

\section{Arterial Spin-Labeling}

In ASL, 2 images are acquired, 1 with and 1 without a labeling pulse. ${ }^{18}$ In both images, blood is allowed a certain delay time to flow into the imaging volume. The difference between the 2 images shows only signal from spins that have moved into the tissue and is therefore a measure of CBF. The delay time ranges between
1 and 3 seconds to allow the blood to reach the capillary bed. In principle, longer delay times would allow the spins to reach the venous system. However, the T1-relaxation time is short relative to the arterial-venous transit time, and the labeling has decayed before spins reach the venous system. The postlabeling delay poses a trade-off. For short delays, the blood may not have reached all brain regions. For long delays, the labeling has decayed due to $\mathrm{T} 1$ relaxation, resulting in a reduced signal-to-noise ratio. Because of the prolonged T1, ASL benefits from higher field strengths. Scan times are between 2 and 4 minutes for this technique. ${ }^{19}$ The recently published consensus guidelines recommend the use of pseudocontinuous ASL labeling. ${ }^{19}$ Due to the lower signal-to-noise ratio of ASL compared with DSC, spatial resolution is sometimes lower than in DSC, further complicating the evaluation of cortical gray matter and small lesions, so ALS is currently not recommended for use in WM. ${ }^{19}$ There are several additional pitfalls with ASL. The labeling may be inefficient, resulting in low apparent CBF in the insufficiently labeled vascular territory. Motion between the 2 scans may be present but can be mitigated by image registration and by suppressing nonmoving spins (ie, background tissue). Areas more distal to the labeling plane have longer transit times during which some of the labeling has decayed. This effect may be erroneously interpreted as reduced CBF.

\section{Perfusion in MS Lesions}

Wuerfel et $\mathrm{al}^{20}$ first analyzed the evolution of the perfusion of gadolinium-enhancing lesion formation in 20 patients with relapsing-remitting multiple sclerosis (RRMS) using spin-echo DSC. They observed a 20\% increase in CBF and CBV from baseline up to 3 weeks before enhancement, a $25 \%$ increase at the time of enhancement, and a slow decline to baseline during 20 weeks after initial gadolinium enhancement. Ring-enhancing lesions showed similar changes only in the enhancing periphery, the site of maximal inflammation on pathology. ${ }^{21}$ Indeed, clustering of macrophages and active demyelination are present at the edge of active MS ring-enhancing lesions. ${ }^{21}$ These findings suggest that perfusion MR imaging is highly sensitive to inflammatory activity and able to show changes long before and after blood-brain barrier disruption can be detected with gadolinium enhancement.

DCE-MR imaging permits observation of enhancement patterns across more time. ${ }^{17}$ Gaitán et $\mathrm{al}^{17}$ found that smaller nodular contrast-enhancing lesions enhance centrifugally, whereas larger ring-enhancing or expanding nodular enhancing lesions enhance centripetally; this feature likely reflects the outward expansion of the lesions from the central vein during their formation.

Observations from cross-sectional studies also suggested that perfusion in lesions is dynamic and related to inflammation. Studies using either gradient-echo DSC or DCE MR imaging found increased $\mathrm{CBV}$ and $\mathrm{CBF}$ in contrast-enhancing lesions compared with normal-appearing white matter (NAWM) (Table). ${ }^{12,22,23}$ However, Ge et $\mathrm{al}^{12}$ found no difference between $\mathrm{CBV}$ and $\mathrm{CBF}$ in contrast-enhancing MS lesions compared with the WM in healthy controls. They also described 2 perfusion patterns in nonenhancing lesions: A subset of these lesions exhibited lower CBF than WM in healthy controls as well as lower CBF and 


\begin{tabular}{lcccc}
\hline & Active Lesions & Chronic Lesions & NAWM & Deep Gray Matter \\
\hline CBF & $\uparrow$ (Compared with NAWM) & $\downarrow$ (Compared with NAWM and & $\downarrow$ (Compared with WM in controls) & $\downarrow$ (Compared with controls) \\
& & WM in controls) & & \\
CBV & $\uparrow$ (Compared with NAWM) & $\downarrow$ (Compared with NAWM) & $\downarrow$ (Compared with WM in controls) & $\downarrow$ (Compared with controls) \\
MTT & No significant change & $\uparrow$ (Compared with WM in & $\uparrow$ (Compared with WM in controls) & $\uparrow$ (Compared with controls) \\
& & controls) & & \\
\hline
\end{tabular}

Note:- $\uparrow$ indicates increased; $\downarrow$, decreased

CBV than NAWM in subjects with MS. The remaining lesions were more like enhancing lesions and presumably also in an inflammatory state, with increased CBV in comparison with MS NAWM but not increased compared with healthy control WM.

On the other hand, T1-hypointense lesions (black holes) were found to have lower CBV values than T1-isointense lesions and healthy control WM..$^{23,24}$ In contrast, T1-isointense lesions did not differ from healthy control WM. ${ }^{24}$ Black holes are characterized by axonal loss and thus are likely to be less perfused and metabolically less active. ${ }^{23,24}$

Cortical lesions were also evaluated using DSC-MR imaging in 44 patients with RRMS. ${ }^{13}$ Most exhibited significantly lower CBF and CBV than normal-appearing gray matter. Nonetheless, like WM lesions, some cortical lesions showed increased CBF and/or $\mathrm{CBV}$, again thought to reflect acute inflammation. Accurate cortex delineation on perfusion imaging can be challenging. However, in that study, double inversion recovery images were registered to DSC, and every voxel was individually analyzed to reduce bias due to noise, partial volume, and nonuniformity. ${ }^{13}$

\section{Perfusion in Normal-Appearing Tissue}

Most cross-sectional studies assessing perfusion in NAWM identified altered perfusion parameters with different combinations of either reduced $\mathrm{CBF}$ or reduced $\mathrm{CBV}$ and $\mathrm{CBF}$ and elevated mean transit time in patients with MS compared with healthy controls. ${ }^{3,8,12,25-28}$ Only 1 report identified an overall increased WM perfusion in 60 patients with MS with mixed phenotypes, but heterogeneous WM perfusion. ${ }^{29}$

In 17 subjects with RRMS, Law et $\mathrm{al}^{3}$ found overall reduced $\mathrm{CBF}$ and prolonged mean transit time but no changes in $\mathrm{CBV}$ in the NAWM of patients compared with controls. Controls but not patients with MS had higher periventricular $\mathrm{CBV}$ and $\mathrm{CBF}$ relative to the adjacent WM. Consistently, evaluation of WM perfusion revealed a decreased CBF in periventricular WM in 12 patients with clinically isolated syndrome compared with controls, whereas patients with early RRMS also had decreased CBF in deep gray matter. ${ }^{30}$ This finding suggests that periventricular WM is affected early and is distinctively susceptible to the changes leading to a perfusion defect. Periventricular WM is vulnerable to microvascular injury, which has been hypothesized to underlie the hypoperfusion. ${ }^{3}$

Decreased perfusion has also been observed in deep and cortical gray matter of patients with MS compared with healthy controls. ${ }^{26,27,29-33}$ In early RRMS, reduced perfusion but no atrophy was described in multiple cortical areas and deep gray matter structures including the thalamus, caudate, putamen, and hippocampus. ${ }^{26,30}$ Moreover, Debernard et $\mathrm{al}^{26}$ found that these findings correlated to visual and verbal memory impairment in 25 patients with early RRMS. Hence, perfusion changes may constitute a clinically relevant biomarker in early MS, especially because they could precede detectable structural atrophy. ${ }^{26,30}$
Using DCE-MR imaging, a more recent study with high projected statistical power based on calculation using means and SDs derived from a Monte Carlo simulation found much lower CBF and $C B V$ values than the aforementioned reports, with no difference in CBF, CBV, or mean transit time between 16 controls and 24 patients with RRMS. ${ }^{34}$ DCE-MR imaging is rarely used to assess perfusion in MS, and the impact of the differences in technical factors compared with DSC-MR imaging remains to be clarified.

\section{Perfusion in MS Subtypes}

Potential differences in perfusion across MS subtypes are predominately based on the small numbers of subjects in each investigation, precluding definitive conclusions.

In 3 early (4- to 5-year disease duration) RRMS cohorts, low perfusion in NAWM and gray matter was reported. ${ }^{26,30,32}$ Two studies in patients with clinically isolated syndrome reported regional CBF decreases in either periventricular NAWM or deep gray matter compared with healthy controls. ${ }^{30,35} \mathrm{CBV}$ and mean transit time were found to be elevated relative to healthy controls in NAWM and deep gray matter in 1 of the 2 studies. ${ }^{35}$ Differences in perfusion between progressive and relapsing-remitting subgroups have not been consistently demonstrated. Patients with primary-progressive MS have been found to have significantly or trending lower perfusion in normal-appearing gray matter and periventricular NAWM than patients with RRMS. ${ }^{8,25,29,31}$ In fact, Adhya et $\mathrm{al}^{25}$ identified significantly lower CBF and CBV in periventricular NAWM and lower CBV in frontal WM, without any significant difference in other WM regions. This finding is in line with the aforementioned observations in clinically isolated syndrome and early RRMS cohorts and implies a unique susceptibility of periventricular WM to declining perfusion in progressive disease as well. Mean cortical gray matter CBF has also been described as lower in secondary-progressive MS than in RRMS in a large MS cohort, but significance was lost after adjusting for other accounting factors such as T2 lesion volume, age, sex, and disease duration. ${ }^{36}$

\section{Clinical Correlations}

Despite compelling and reproducible perfusion abnormalities in MS, their relation to clinical measures of disability and disease severity has not been strongly established. No association between the Expanded Disability Status Scale or disease duration and perfusion parameters in NAWM or gray matter has been identified in clinically isolated syndrome and early RRMS cohorts, or in most mixed MS phenotype cohorts. ${ }^{8,23,26,27,29-31,33,35}$ Two studies revealed a negative correlation between the Expanded Disability Status Scale and CBF and CBV in NAWM. ${ }^{25,37}$ One study of 23 subjects with RRMS found a significant negative association between hypoperfusion in the nonatrophic bilateral median thalami 
and time to complete the 9-Hole Peg Test. ${ }^{32}$ No correlation between perfusion in white matter lesions and disability has been found, though these were assessed in only 1 article. ${ }^{23}$ Likewise, although they were evaluated in most publications, relationships between perfusion measurements and either T2 or T1 or contrastenhancing lesion volumes or white and gray matter atrophy were not identified in most reports. ${ }^{8,25,26,30,31,35,37}$

The absence of a correlation between perfusion alterations and gross measures of disability may be due to the lack of sensitivity of these outcome measures to predict disability. ${ }^{38}$ The Expanded Disability Status Scale mostly reflects gait disability related to spinal cord disease and has been criticized for its variability among examiners. ${ }^{38}$ The 9-Hole Peg Test reliably detects progression in patients with MS but is a measure specific to upper extremity motor function. ${ }^{39}$ Moreover, MR imaging gadolinium-enhancing or T2 lesion load is not a good predictor of long-term disability. ${ }^{38}$ Nevertheless, the usefulness of perfusion MR imaging probably lies more in its ability to detect acute inflammatory changes earlier than conventional MR imaging as detailed above. ${ }^{20}$ This ability could lead to earlier identification of symptomatic and asymptomatic disease activity underlying relapses and new T2 lesions, which are known to impact disability. ${ }^{38}$

D'haeseleer et $\mathrm{al}^{40}$ found that Paced Auditory Serial Addition Test scores in 10 patients with RRMS and 10 with primary-progressive MS correlated strongly with reduced CBF in the left centrum semiovale, an area involved in processing speed and verbal working memory. In an early RRMS cohort, visual and verbal memory impairment was related to perfusion reduction in many cortical and deep gray matter areas. ${ }^{26}$ Moreover, Inglese et $\mathrm{al}^{8}$ reported a moderate-to-strong positive association between deep gray matter CBF and visuoperceptual ability in 18 patients with RRMS and 14 with primary-progressive MS. They also measured inhibition switching, which was also moderately correlated to deep gray matter CBV. The same group identified Multidimensional Fatigue inventory reduced activity and reduced motivation scores as correlates to deep gray matter perfusion. ${ }^{31}$ Furthermore, perfusion analysis in 25 cognitively impaired versus 20 unimpaired subjects with secondary-progressive MS revealed, in the former, lower quantitative CBV in the bilateral superior medial frontal cortex and lower quantitative CBV and CBF in the thalami and caudate nuclei. ${ }^{41}$ Overall impairment in the Minimal Assessment of Cognitive Function battery as well as subscores of processing speed, verbal fluency, and visuospatial memory showed a moderate positive correlation with these perfusion findings. It is unknown whether a common factor alters both perfusion and cognition or whether one influences the other, but decreased brain perfusion could be a marker of cognitive impairment and fatigue in MS, 2 prevalent and disabling symptoms. ${ }^{42}$ However, the impact of perfusion normalization on cognitive dysfunction in MS is still to be assessed.

\section{Limitations to the Interpretation of the Data}

Interpretation of the summarized data is limited by several factors. First, although longitudinal studies would provide valuable data on how the perfusion characteristics of brain tissue change due to MS, only 1 study assessed changes in perfusion across time. ${ }^{20}$ There is evidence that contrast agents accumulate in the brain $^{43}$; this finding hampers their repeat administration in longitudinal studies using DSC. Furthermore, the ill-posed deconvolution and the selection of the arterial input function required in DSC make serial studies particularly difficult to perform. ${ }^{9}$

Second, analysis of perfusion imaging requires many technical steps that are each susceptible to measurement error. Imaging protocols and techniques are also heterogeneous, with most studies using DSC-MR imaging but some using ASL. ${ }^{26-29,32,33,36,40,44}$ As explained above, ASL is not recommended to evaluate WM because of its low signal-to-noise ratio. ${ }^{19}$ Delineation of ROIs also varies greatly, from specific gyri to global white or gray matter. The spatial resolution of perfusion MR images, typically 8-27 $\mathrm{mm}^{3}$, does not always allow a clear distinction between gray and white matter, making accurate cortex delineation and evaluation of MS lesions with diameters of $<3-4 \mathrm{~mm}$ challenging. External factors, such as the degree of hydration and substances such as caffeine or ethanol, can also influence cerebral perfusion ${ }^{45-47}$ but have not been accounted for in any MS perfusion study. Furthermore, although technical factors can alter the comparison among subjects, analysis of lesion perfusion has been performed using NAWM as a comparator, which is known to be abnormal in MS.

There is also an issue with using any area of the brain to normalize perfusion maps. Indeed, CBF maps obtained after deconvolution are dependent on the arterial input function, which is influenced by contrast agent properties, partial volume effects, and bolus delay and dispersion. ${ }^{48}$ A way to reduce the contribution of these artifacts is to normalize the values using a reference area of the brain, but this is rarely done. ${ }^{35}$ Normalization however introduces another potential bias because no part of the brain is entirely normal at a pathological level in the MS brain. ${ }^{49}$

Additionally, it has been recently demonstrated that gradientecho DSC perfusion measurements are strongly influenced by the anisotropic nature of the WM vasculature, with angle-dependent variations of up to $130 \%$ in $\mathrm{CBF}$ and $\mathrm{CBV} .{ }^{50}$ This orientation dependency is caused by blood vessels running in parallel to WM tracts, the effect being much weaker for vessels parallel to the main magnetic field than for vessels perpendicular to the main magnetic field. Both CBF and CBV in lesions that are traversed by a vein parallel to the orientation of the main magnetic field, such as Dawson fingers, may appear lower than in lesions traversed by a vein perpendicular to the main magnetic field. ${ }^{50}$ To our knowledge, tissue orientation was not accounted for in any of the previous studies. For studies comparing nonlesional tissue among groups on the other hand, this limitation may be less relevant. Moreover, spin-echo DSC is much less affected by orientation effects, ${ }^{51}$ but gradient-echo was used in all the aforementioned DSC assessments. Gradient-echo DSC also has signal drop-out near tissue-air and tissue-bone interfaces, making these brain areas difficult to evaluate. ${ }^{52}$

Third, groups of subjects are widely heterogeneous in their disease duration, disability, presence or absence of MS disease-

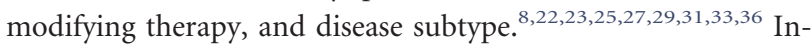
flammation is generally less prominent with longer disease duration and in progressive forms of $\mathrm{MS}^{21}$ Disease-modifying therapies can also reduce inflammation. ${ }^{1}$ Consequently, these factors are likely to influence perfusion, with lower brain CBF and $\mathrm{CBV}$ in less inflammatory conditions, such as in progressive dis- 
ease $^{8,25,29,31}$ and as evidenced by higher NAWM CBV and CBF in patients with MS with repeat new contrast-enhancing lesions. ${ }^{37}$

\section{Hypotheses Underlying Perfusion Modifications}

Increased perfusion in active lesions most likely represents vasodilation secondary to inflammation of the involved gray or white matter. ${ }^{20}$ More uncertainties surround the explanation for the diffuse perfusion decrease in NAWM and gray matter, a phenomenon attributed to either a primary vascular insult or reduced tissue metabolic function from neuronal loss. ${ }^{53}$ Evidence of cerebral vascular dysfunction has been brought forward in MS but is not sufficient to explain alterations of brain perfusion. A combination of factors is more likely at play.

\section{Vascular Involvement}

Initially, the controversial and now refuted theory of chronic cerebrospinal vascular insufficiency underlying MS caused a lot of attention. Nonetheless, epidemiologic studies have identified a small excess of strokes in patients with MS, ${ }^{54}$ though lifestyle factors such as smoking were not accounted for. Moreover, some pathologic findings support vascular involvement in MS. ${ }^{3,4}$ It is well-accepted that plaques occur around a central vein and DCE-MR imaging findings support the formation and expansion of newly formed lesions around that central vein. ${ }^{2,17}$ Vasculitic changes, reflected by lymphocytic infiltration of the vein walls, have been hypothesized to predate plaque development. ${ }^{3}$ Indeed, type III demyelinating MS lesions have pathologic findings common to WM lesions occurring on an ischemic background. ${ }^{49,55}$ There is preferential loss of myelin-associated glycoprotein and oligodendrocyte apoptosis with preservation of other myelin proteins. ${ }^{49,55}$ Upregulation of hypoxia inducible factor- $1 \alpha$, a transcription factor involved in response to hypoxia, is present in type III lesion glial cells, ${ }^{49}$ and expression of hypoxia inducible factor- $1 \alpha$ and its downstream genes has been found to be enhanced in secondary-progressive MS. ${ }^{49,53,55}$ However, this gene is also upregulated in states of nonhypoxic energy failure, such as decreased mitochondrial adenosine triphosphate production, ${ }^{55}$ which occurs in MS.

Marshall et $\mathrm{al}^{44}$ have found CBF modulation in gray matter to be less responsive to hypercapnia in subjects with MS than in controls, suggesting dysfunction of cerebrovascular reactivity in MS. This lack of cerebrovascular reactivity was correlated to WM lesion volume and global gray matter atrophy. ${ }^{38}$ Dysfunctional vasoactivity of the cerebral vasculature has been further evidenced by elevated jugular levels of endothelin-1, a potent vasoconstrictor, measured in 15 patients with MS compared with controls. ${ }^{56}$ An increase of their CBF by $20 \%$ after administration of bosentan, an endothelin-1 antagonist, confirmed the mechanistic impact of endothelin-1 on brain perfusion and suggested potential reversibility of CBF impairment.

Despite evidence of vascular dysfunction in MS, it is unclear whether brain damage results from or engenders the perfusion defect. Looking at perfusion and diffusion tensor imaging in a small cohort of patients with RRMS, Saindane et $\mathrm{al}^{57}$ found that decreasing $\mathrm{CBF}$ and $\mathrm{CBV}$ in the corpus callosum significantly correlated with decreasing mean diffusivity but not fractional anisotropy. This pattern was recognized as consistent with a primary ischemic insult rather than hypoperfusion resulting from axonal degeneration, which would translate into increased mean diffusivity and decreased fractional anisotropy. ${ }^{57}$ However, alternative explanations, namely a reduced axonal energy state without irreversible axonal degeneration, are not excluded. Some findings suggest that reduced perfusion could limit repair and remyelination. In fact, chronic T2 lesions and T1-hypointense lesions seem to occur predominantly in areas of reduced CBF, as indicated by perfusion maps on imaging ${ }^{28,58}$ or anatomic watershed areas on pathology. ${ }^{59}$ Moreover, in a very large MS cohort, Holland et a ${ }^{58}$ found that preferential occurrence of lesions in areas of low perfusion was more frequent in secondary-progressive MS than in RRMS.

Certain observations argue against a primary vascular pathology in MS. ${ }^{49}$ The increased perfusion observed in acute lesions indicates inflammation rather than primary ischemia. The diffuse rather than regional abnormal perfusion is also not in agreement with presumed focal vasculitic involvement. In addition, microvessel thrombosis is only rarely identified on pathology, and MS-like lesions are not typically induced by stroke, the prototypical example of brain ischemia. ${ }^{49}$

\section{Metabolic Dysfunction}

Neuronal loss has been hypothesized to explain the diffuse reduction in perfusion in MS by reducing the demand for energy. ${ }^{26}$ Nonetheless, the presence of a tissue perfusion decrease despite the absence of atrophy, as described above, argues against this sole mechanism. ${ }^{26}$ Oxidative stress and deficient energy production resulting from mitochondrial dysfunction are thought to be important factors behind neurodegeneration in MS. ${ }^{60}$ The mechanisms leading to mitochondrial injury following demyelination are not fully understood. ${ }^{60}$ Virtual hypoxia refers to reduced adenosine triphosphate production secondary to mitochondrial dysfunction coupled with increased energy demand in demyelinated axons. Virtual hypoxia could lead to axon necrosis. ${ }^{60}$ This metabolic failure could be reflected through reduced perfusion in noninflammatory tissue without actual ischemia. On the other hand, decoupling between the $\mathrm{N}$-acetylaspartate-to-creatine ratio, reflecting axonal metabolism, and $\mathrm{CBF}$ has been observed in the corpus callosum of 25 patients with MS and suggested that metabolic dysfunction or axonal loss alone is unlikely to account for the perfusion modifications. ${ }^{27}$ Alternatively, reduced energy production by astrocytes in MS could contribute to reduced demand in blood flow, which would also manifest as reduced perfusion on MR imaging. ${ }^{49}$ Astrocytes in MS lesions lack $\beta 2$-adrenergic receptors. Norepinephrine-mediated activation of these receptors is important, notably for energy production and vasodilation; default of this pathway would thus lead to reduced $\mathrm{CBF}^{49}$

\section{Conclusions and Future Directions}

There is a large body of limited-quality-but-concordant evidence demonstrating alteration of cerebral perfusion in MS. Perfusion MR imaging appears to be a sensitive tool, perhaps more than conventional MR imaging, for detecting focal inflammatory activity in the brain. Its usefulness to improve early detection of relapses and new lesions and the impact on treatment decision- 
making should be clarified. The effect of disease-modifying therapy on brain perfusion should also be characterized. This characterization would allow assessment of the potential role of perfusion MR imaging as a marker of treatment response through monitoring of inflammatory activity. Diffuse hypoperfusion of white and gray matter is found and could be a clinically relevant marker of cognitive dysfunction and fatigue. The potential for reliable identification of cognitively impaired patients or patients at risk of cognitive dysfunction should be explored. Furthermore, defining the impact of reversing normal-appearing tissue hypoperfusion on cognition and disability could lead to a better understanding of the disease pathogenesis and potentially to novel treatment options for symptoms. Alteration of cerebral perfusion in patients with MS compared with controls certainly raises questions about the pathogenesis of the disease. Reduction of energy demand and blood supply resulting from mitochondrial and astrocytic dysfunction as well as from axonal degeneration perhaps does not fully explain the diffuse reduction in perfusion. Some evidence of hypoxic-mediated injury in MS exists, and dysfunction of cerebrovascular vasoactivity could contribute to hypoperfusion. However, a combination of inflammatory, metabolic, and vascular factors is more likely than a sole primary vascular pathology. Advanced techniques, such as the measurement of the cerebral metabolic rate of oxygen, along with the oxygen extraction fraction, may shed further light on these issues. ${ }^{61}$

Disclosures: Emmanuelle Lapointe-UNRELATED: Consultancy: EMD Serono and Hoffmann-La Roche. Anthony L. Traboulsee-UNRELATED: Grant: Canadian Institute for Health Research*; UNRELATED: Consultancy: Biogen Idec, Hoffman-La Roche, Sanofi Genzyme, Novartis, Teva Canada Innovation; Grants/Grants Pending: MS Society of Canada, Chugai Pharmaceutical Co, Biogen Idec, Sanofi Genzyme, Hoffman-La Roche*; Payment for Lectures Including Service on Speakers Bureaus: Sanofi Genzyme, Hoffman-La Roche; Travel/Accommodations/Meeting Expenses Unrelated to Activities Listed: Sanofi Genzyme. Alexander Rauscher-UNRELATED: Payment for Lectures Including Service on Speakers Bureaus: Philips Healthcare, Comments: fees for a keynote lecture at a Philips MRI Lunch Symposia. *Money paid to the institution.

\section{REFERENCES}

1. Grigoriadis N, van Pesch V, Paradig MS; Paradig MS Group. A basic overview of multiple sclerosis immunopathology. Eur J Neurol 2015;22(suppl 2):3-13 CrossRef Medline

2. Sati $\mathrm{P}, \mathrm{Oh} \mathrm{J}$, Constable RT, et al. The central vein sign and its clinical evaluation for the diagnosis of multiple sclerosis: a consensus statement from the North American Imaging in Multiple Sclerosis Cooperative. Nat Rev Neurol 2016;12:714-22 CrossRef Medline

3. Law M, Saindaine AM, Ge Y, et al. Microvascular abnormality in relapsing-remitting multiple sclerosis: perfusion MR imaging findings in normal-appearing white matter. Radiology 2004;231:645-52 CrossRef Medline

4. D'haeseleer M, Hostenbach S, Peeters I, et al. Cerebral hypoperfusion: a new pathophysiologic concept in multiple sclerosis? J Cereb Blood Flow Metab 2015;35:1406-10 CrossRef Medline

5. Sun X, Tanaka M, Kondo S, et al. Clinical significance of reduced cerebral metabolism in multiple sclerosis: a combined PET and MRI study. Ann Nucl Med 1998;12:89-94 CrossRef Medline

6. Lycke J, Wikkelsö C, Bergh AC, et al. Regional cerebral blood flow in multiple sclerosis measured by single photon emission tomography with technetium-99m hexamethylpropyleneamine oxime. Eur Neurol 1993;33:163-67 Medline

7. Brooks DJ, Leenders KL, Head G, et al. Studies on regional cerebral oxygen utilisation and cognitive function in multiple sclerosis. J Neurol Neurosurg Psychiatry 1984;47:1182-91 CrossRef Medline

8. Inglese M, Adhya S, Johnson G, et al. Perfusion magnetic reso- nance imaging correlates of neuropsychological impairment in multiple sclerosis. J Cereb Blood Flow Metab 2008;28:164-71 CrossRef Medline

9. MacDonald ME, Frayne R. Cerebrovascular MRI: a review of stateof-the-art approaches, methods and techniques. NMR Biomed 2015; 28:767-91 CrossRef Medline

10. Boxerman JL, Hamberg LM, Rosen BR, et al. MR contrast due to intravascular magnetic susceptibility perturbations. Magn Reson Med 1995;34:555-66 CrossRef Medline

11. Weisskoff RM, Zuo CS, Boxerman JL, et al. Microscopic susceptibility variation and transverse relaxation: theory and experiment. Magn Reson Med 1994;31:601-10 CrossRef Medline

12. Ge Y, Law M, Johnson G, et al. Dynamic susceptibility contrast perfusion MR imaging of multiple sclerosis lesions: characterizing hemodynamic impairment and inflammatory activity. AJNR Am J Neuroradiol 2005;26:1539-47 Medline

13. Peruzzo D, Castellaro M, Calabrese M, et al. Heterogeneity of cortical lesions in multiple sclerosis: an MRI perfusion study. J Cereb Blood Flow Metab 2013;33:457-63 CrossRef Medline

14. Barth M, Breuer F, Koopmans PJ, et al. Simultaneous multislice (SMS) imaging techniques. Magn Reson Med 2016;75:63-81 CrossRef Medline

15. Todd N, Moeller S, Auerbach EJ, et al. Evaluation of 2D multiband EPI imaging for high-resolution, whole-brain, task-based fMRI studies at 3T: sensitivity and slice leakage artifacts. Neuroimage 2016;124(Pt A):32-42 CrossRef Medline

16. Tofts PS, Brix G, Buckley DL, et al. Estimating kinetic parameters from dynamic contrast-enhanced T(1)-weighted MRI of a diffusable tracer: standardized quantities and symbols. J Magn Reson Imaging 1999;10:223-32 Medline

17. Gaitán MI, Shea CD, Evangelou IE, et al. Evolution of the bloodbrain barrier in newly forming multiple sclerosis lesions. Ann Neurol 2011;70:22-29 CrossRef Medline

18. Williams DS, Detre JA, Leigh JS, et al. Magnetic resonance imaging of perfusion using spin inversion of arterial water. Proc Natl Acad Sci US A 1992;89:212-16 CrossRef Medline

19. Alsop DC, Detre JA, Golay X, et al. Recommended implementation of arterial spin-labeled perfusion MRI for clinical applications: a consensus of the ISMRM perfusion study group and the European consortium for ASL in dementia. Magn Reson Med 2015;73:102-16 CrossRef Medline

20. Wuerfel J, Bellmann-Strobl J, Brunecker P, et al. Changes in cerebral perfusion precede plaque formation in multiple sclerosis: a longitudinal perfusion MRI study. Brain 2004;127(Pt 1):111-19 CrossRef Medline

21. Lassmann $H$. The pathologic substrate of magnetic resonance alterations in multiple sclerosis. Neuroimaging Clin N Am 2008;18:56376, ix CrossRef Medline

22. Ingrisch M, Sourbron S, Morhard D, et al. Quantification of perfusion and permeability in multiple sclerosis: dynamic contrast-enhanced MRI in 3D at 3T. Invest Radiol 2012;47:252-58 CrossRef Medline

23. Haselhorst R, Kappos L, Bilecen D, et al. Dynamic susceptibility contrast MR imaging of plaque development in multiple sclerosis: application of an extended blood-brain barrier leakage correction. $J$ Magn Reson Imaging 2000;11:495-505 Medline

24. Li L, Chopp M, Nejad-Davarani SP, et al. Perfusion and diffusion abnormalities of multiple sclerosis lesions and relevance of classified lesions to disease status. J Neurol Neurophysiol 2014;2014(Suppl 12):12 CrossRef Medline

25. Adhya S, Johnson G, Herbert J, et al. Pattern of hemodynamic impairment in multiple sclerosis: dynamic susceptibility contrast perfusion MR imaging at 3.0 T. Neuroimage 2006;33:1029-35 CrossRef Medline

26. Debernard L, Melzer TR, Van Stockum S, et al. Reduced grey matter perfusion without volume loss in early relapsing-remitting multiple sclerosis. J Neurol Neurosurg Psychiary 2014;85:544-51 CrossRef Medline

27. Steen C, D'haeseleer M, Hoogduin JM, et al. Cerebral white matter 
blood flow and energy metabolism in multiple sclerosis. Mult Scler 2013;19:1282-89 CrossRef Medline

28. Narayana PA, Zhou Y, Hasan KM, et al. Hypoperfusion and T1hypointense lesions in white matter in multiple sclerosis. Mult Scler 2014;20:365-73 CrossRef Medline

29. Rashid W, Parkes LM, Ingle GT, et al. Abnormalities of cerebral perfusion in multiple sclerosis. J Neurol Neurosurg Psychiary 2004; 75:1288-93 CrossRef Medline

30. Varga AW, Johnson G, Babb JS, et al. White matter hemodynamic abnormalities precede sub-cortical gray matter changes in multiple sclerosis. J Neurol Sci 2009;282:28-33 CrossRef Medline

31. Inglese $M$, Park SJ, Johnson $G$, et al. Deep gray matter perfusion in multiple sclerosis: dynamic susceptibility contrast perfusion magnetic resonance imaging at 3 T. Arch Neurol 2007;64:196-202 CrossRef Medline

32. Doche E, Lecocq A, Maarouf A, et al. Hypoperfusion of the thalamus is associated with disability in relapsing remitting multiple sclerosis. J Neuroradiol 2017;44:158-64 CrossRef Medline

33. Ota M, Sato N, Nakata Y, et al. Abnormalities of cerebral blood flow in multiple sclerosis: a pseudocontinuous arterial spin labeling MRI study. Magn Reson Imaging 2013;31:990-95 CrossRef Medline

34. Ingrisch $\mathrm{M}$, Sourbron $\mathrm{S}$, Herberich $\mathrm{S}$, et al. Dynamic contrast-enhanced magnetic resonance imaging suggests normal perfusion in normal-appearing white matter in multiple sclerosis. Invest Radiol 2017;52:135-41 CrossRef Medline

35. Papadaki EZ, Mastorodemos VC, Amanakis EZ, et al. White matter and deep gray matter hemodynamic changes in multiple sclerosis patients with clinically isolated syndrome. Magn Reson Med 2012; 68:1932-42 CrossRef Medline

36. Amann M, Achtnichts L, Hirsch JG, et al. 3D GRASE arterial spin labelling reveals an inverse correlation of cortical perfusion with the white matter lesion volume in MS. Mult Scler 2012;18:1570-76 CrossRef Medline

37. Bester M, Forkert ND, Stellmann JP, et al. Increased perfusion in normal appearing white matter in high inflammatory multiple sclerosis patients. PLoS One 2015;10:e0119356 CrossRef Medline

38. Hyland M, Rudick RA. Challenges to clinical trials in multiple sclerosis: outcome measures in the era of disease-modifying drugs. Curr Opin Neurol 2011;24:255-61 CrossRef Medline

39. Feys P, Lamers I, Francis G, et al; Multiple Sclerosis Outcome Assessments Consortium. The Nine-Hole Peg Test as a manual dexterity performance measure for multiple sclerosis. Mult Scler 2017;23: 711-20 CrossRef Medline

40. D'haeseleer M, Steen C, Hoogduin JM, et al. Performance on Paced Auditory Serial Addition Test and cerebral blood flow in multiple sclerosis. Acta Neurol Scand 2013;128:e26-29 CrossRef Medline

41. Francis PL, Jakubovic R, O'Connor P, et al. Robust perfusion deficits in cognitively impaired patients with secondary-progressive multiple sclerosis. AJNR Am J Neuroradiol 2013;34:62-67 CrossRef Medline

42. Penner IK. Evaluation of cognition and fatigue in multiple sclerosis: daily practice and future directions. Acta Neurol Scand 2016; 134(suppl 200):19-23 CrossRef Medline

43. Ramalho J, Semelka RC, Ramalho M, et al. Gadolinium-based contrast agent accumulation and toxicity: an update. AJNR Am J Neuroradiol 2016;37:1192-98 CrossRef Medline

44. Marshall $\mathrm{O}, \mathrm{Lu} \mathrm{H}$, Brisset JC, et al. Impaired cerebrovascular reactivity in multiple sclerosis. JAMA Neurol 2014;71:1275-81 CrossRef Medline

45. Field AS, Laurienti PJ, Yen YF, et al. Dietary caffeine consumption and withdrawal: confounding variables in quantitative cerebral perfusion studies? Radiology 2003;227:129-35 CrossRef Medline

46. Trangmar SJ, Chiesa ST, Llodio I, et al. Dehydration accelerates reductions in cerebral blood flow during prolonged exercise in the heat without compromising brain metabolism. Am J Physiol Heart Circ Physiol 2015;309:598-607 CrossRef Medline

47. Mathew RJ, Wilson WH. Substance abuse and cerebral blood flow. Am J Psychiatry 1991;148:292-305 CrossRef Medline

48. Calamante F. Arterial input function in perfusion MRI: a comprehensive review. Prog Nucl Magn Reson Spectrosc 2013;74:1-32 CrossRef Medline

49. De Keyser J, Steen C, Mostert JP, et al. Hypoperfusion of the cerebral white matter in multiple sclerosis: possible mechanisms and pathophysiological significance. J Cereb Blood Flow Metab 2008;28: 1645-51 CrossRef Medline

50. Hernández-Torres E, Kassner N, Forkert ND, et al. Anisotropic cerebral vascular architecture causes orientation dependency in cerebral blood flow and volume measured with dynamic susceptibility contrast magnetic resonance imaging. J Cereb Blood Flow Metab 2017;37:1108-19 CrossRef Medline

51. Doucette J, Wei L, Kames C, et al. Anisotropic cerebral vascular architecture causes orientation dependency in cerebral blood flow and volume measured with spin echo dynamic susceptibility contrast magnetic resonance imaging. In: Proceedings of the Annual Meeting of the International Society for Magnetic Resonance in Medicine, Honolulu, Hawaii. April 22-27, 2017

52. Deichmann R, Gottfried J, Hutton C, et al. Optimized EPI for fMRI studies of the orbitofrontal cortex. Neuroimage 2003;19(2 Pt 1): 430-41 CrossRef Medline

53. D'haeseleer M, Cambron M, Vanopdenbosch L, et al. Vascular aspects of multiple sclerosis. Lancet Neurol 2011;10:657-66 CrossRef Medline

54. Christiansen CF, Christensen S, Farkas DK, et al. Risk of arterial cardiovascular diseases in patients with multiple sclerosis: a populationbased cohort study. Neuroepidemiology 2010;35:267-74 CrossRef Medline

55. Aboul-Enein F, Rauschka H, Kornek B, et al. Preferential loss of myelin-associated glycoprotein reflects hypoxia-like white matter damage in stroke and inflammatory brain diseases. J Neuropathol Exp Neurol 2003;62:25-33 CrossRef Medline

56. D'haeseleer M, Beelen R, Fierens Y, et al. Cerebral hypoperfusion in multiple sclerosis is reversible and mediated by endothelin-1. Proc Natl Acad Sci U S A 2013;110:5654-58 CrossRef Medline

57. Saindane AM, Law M, Ge Y, et al. Correlation of diffusion tensor and dynamic perfusion MR imaging metrics in normal-appearing corpus callosum: support for primary hypoperfusion in multiple sclerosis. AJNR Am J Neuroradiol 2007;28:767-72 Medline

58. Holland CM, Charil A, Csapo I, et al. The relationship between normal cerebral perfusion patterns and white matter lesion distribution in 1,249 patients with multiple sclerosis. J Neuroimaging 2012; 22:129-36 CrossRef Medline

59. Haider L, Zrzavy T, Hametner S, et al. The topograpy of demyelination and neurodegeneration in the multiple sclerosis brain. Brain 2016;139(Pt 3):807-15 CrossRef Medline

60. Mahad DH, Trapp BD, Lassmann H. Pathological mechanisms in progressive multiple sclerosis. Lancet Neurol 2015;14:183-93 CrossRef Medline

61. Wise RG, Harris AD, Stone AJ, et al. Measurement of OEF and absolute CMRO2: MRI-based methods using interleaved and combined hypercapnia and hyperoxia. Neuroimage 2013;83:135-47 CrossRef Medline 